\title{
Oil Solubilization and Aggregation Behavior of Cetyl Trimethyl Ammonium Bromide Surfactant and its Derivatives by SANS
}

\author{
MAHENDRA S BORSE ${ }^{1 *}$, TRYAMBAKRAO J PATIL ${ }^{2}$ and SACHIN S BORSE ${ }^{3}$ \\ ${ }^{1}$ Department of Chemistry, Faculty of Science, Uttamrao Patil College, Dahivel, \\ Sakri - 424304, Maharashtra, India \\ ${ }^{2}$ Department of Chemistry, JET's Z. B. Patil College, Dhule, Maharashtra, India \\ ${ }^{3}$ Department of Chemistry, V. U. Patil Arts and Dr. B. S. Desale Science College, \\ Sakri -424304, Maharashtra, India \\ mahendraborse@yahoo.com
}

Received 17 April 2014 / Accepted 2 May 2014

\begin{abstract}
To understand the role of molecular architecture of surfactant molecule on micellar structure, a series of cationic surfactants was taken viz. cetyl trimethyl ammonium bromide (CTAB-1), cetyl hydroxyethyldimethyl ammonium bromide (CTAB-2) and cetyl dihydroxyethyl methyl ammonium bromide (CTAB-3) has been investigated by small angle neuron scattering measurement. Critical micelle concentration, Kraft temperature for a series of surfactant was determined by conductance measurement, where as aggregation number, effective fractional charge per monomer, equilibrium distance between the charged heads and dimension of micelle were determined from small angle neutron scattering data. It is observed that the number density of micelle and dimension of micelle were observed to increase and hence oil solubilization of surfactant system was also observed to increase from CTAB-1 to CTAB-2 and then CTAB-3.
\end{abstract}

Keywords: CTAB, SANS, Micelle, Oil solubilization, Surfactant

\section{Introduction}

Surfactants, sometimes called surface-active agents or detergents, are extremely versatile chemicals with applications in chemistry, biology and pharmaceutical science ${ }^{1-2}$. Surfactants contain both a non-polar long-chain hydrocarbon "tail" and a polar "head" group called as amphiphilic character. This amphiphilic character of surfactants allows for self-association or micellization, whereby the hydrophobic portion forms the micelle core and the polar head groups form the micelle-water-interface. Surfactant molecules above critical micellar concentration (CMC) in aqueous solution are known to form variety of microstructures such as spherical, ellipsoidal, vesicular, rod-like and thread-like $\mathrm{e}^{3-5}$. The microstructure of surfactant aggregates depends on the molecular architecture of the surfactant and solution conditions, 
such as concentration and temperature ${ }^{6-8}$. The structures of micelles (sizes and shapes) in a variety of micellar systems have been studied using small angle neutron scattering (SANS) study ${ }^{9-11}$. Cationic surfactants belongs to class of quaternary salts are well known compounds and have been examined for their surface and solution behavior using variety of methods ${ }^{12-16}$. The fundamental property of surfactants is their ability to adsorb at interface and forms micelle in solution. This property is due to the presence of both hydrophilic and hydrophobic groups in each surfactant molecules and it is characterized by hydrophilic lipophilic balance (HLB) value $^{17-19}$. It is very interesting that to tune the HLB value of surfactant molecule by changing the molecular architecture will significantly influence surfactant behavior.

Hence a systematic study has been undertaken to study the effect of molecular architecture (head group polarity) of cetyl trimethyl ammonium bromide surfactant and its derivatives on their micelle behavior by SANS. The chemical structures of the surfactants under study are illustrated in Figure 1.

a) Cetyl trimethyl ammonium bromide (CTAB-1)

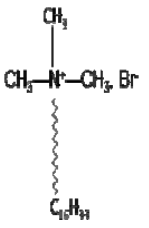

b) Cetyl hydroxyethyl dimethyl ammonium bromide (CTAB-2)

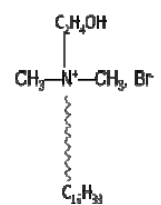

c) Cetyl dihydroxyethyl methyl ammonium bromide (CTAB-3)

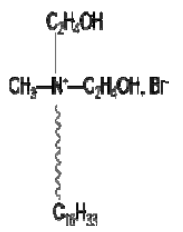

Figure 1. Nomenclature, abbreviations and structures of cationic surfactants under study.

\section{Experimental}

Cetyl trimethyl ammonium bromide was purched from Aldrich and its derivatives viz cetyl hydroxyethyldimethyl ammonium bromide and cetyl dihydroxyethylmethyl ammonium bromide were synthesized and purified by well known method ${ }^{20}$. The purity and structure of synthesized surfactant molecules were checked by FTIR and ${ }^{1} \mathrm{H}$ NMR. Solutions for SANS studies were prepared in $\mathrm{D}_{2} \mathrm{O}$ (at least $99 \%$ D pure) obtained from the Heavy Water Division, Bhabha Atomic Research Center (BARC), Mumbai, India. Double-distilled and deionized water was used for all physicochemical studies.

\section{Conductance measurement}

The electrical conductance of different solutions as a function of concentration was measured using Digital Conductivity Meter EQ-664 (Equiptronic, Mumbai, India) with cell 
constant $1.01 \mathrm{~cm}^{-1}$ at $30.0 \pm 0.1{ }^{\circ} \mathrm{C}$. The average degree of micelle ionization $\left(\alpha_{\text {ave }}\right)$ of the micelle and $\mathrm{CMC}$ were determined from conductivity data.

\section{Kraft temperature}

The Kraft temperature $\left(\mathrm{k}_{\mathrm{T}}\right)$ for a series of surfactants CTAB-1, CTAB-2 and CTAB-3 were determined through conductance measurements as well as visual observation of the transparency of the system. The measurements were repeated at least three times and reproducibility in $\mathrm{k}_{\mathrm{T}}$ values was observed within $\pm 0.2{ }^{\circ} \mathrm{C}$.

\section{Foamability and foam stability}

Foamability and foam stability for series of surfactant systems were studied as per the method reported by Shah and coworker ${ }^{21-22}$. A graduated glass cylinder of $100 \mathrm{~cm}^{3}$ volume was used for the measurement of the foam stability and foamability. Twenty cubic centimeters surfactants solution was poured into the calibrated cylinder. The solution was given 10 uniform jerks within $10 \mathrm{~s}$. The volume of the foam generated was measured as foamability and the time required for the collapse of the foam to half of its initial height was taken as a measure for the foam stability. The experiments were repeated at least five times.

\section{Oil solubilization capacity}

A series of surfactants (CTAB-1, CTAB-2 and CTAB-3) solutions of volume $5 \mathrm{~mL}$, having concentration $0.1 \mathrm{M}$ was prepared. These Solutions were thoroughly homogenized using a vortex mixter and kept in a thermostated water bath at $30 \pm 0.1{ }^{\circ} \mathrm{C}$. These solution were then titrated with methyl methacrylate (oil) using a micro-burette.

\section{Small-angle neutron-scattering (SANS) measurements}

All solutions used in SANS studies were prepared in $\mathrm{D}_{2} \mathrm{O}$ (at least $99 \% \mathrm{D}$ ). This provides a good contrast between the micelles and the solvent in SANS experiment. Neutron scattering measurements were carried out on the SANS Diffractometer at Dhruva Reactor, Trombay, Mumbai, India using a $\mathrm{BeO}$ filtered beam of $15 \%$ resolution $(\Delta \mathrm{Q} / \mathrm{Q})$ at $\mathrm{Q}=0.05 \AA^{-1}$. The sample to detector distance was $1.8 \mathrm{~m}$ for all the runs. The angular distribution of the scattered neutrons was recorded using position sensitive detector (PSD). The accessible wave vector transfer $(\mathrm{Q})$ is given as $4 \pi \sin \theta / \lambda$, where $\lambda$ is the wavelength of the incident neutron and $2 \theta$ is the scattering angle. Range of $Q$ for the instrument lies between 0.02 and $0.30 \AA^{-1}$. PSD allows a simultaneous recording of the data over the full $\mathrm{Q}$ range. The wavelength $(\lambda)$ used in measurement was $5.2 \AA$. The solutions were held in a $0.5 \mathrm{~cm}$ path length UV grade quartz sample holder with tight-fitting Teflon stopper sealed with parafilm. The scattering intensities from the surfactant solutions were corrected for detector background sensitivity, empty cell scattering and sample transmission. Scattering intensity of solvent was subtracted from that of the sample. The resulting corrected intensities were normalized to absolute cross section units and thus $\mathrm{d} \Sigma / \mathrm{d} \Omega$ versus $\mathrm{Q}$ was obtained (Figure 4 ). This absolute calibration has an estimated uncertainty of $10 \%$. The experimental values were fitted using nonlinear least-square method.

\section{Results and Discussion}

\section{Kraft temperature $\left(k_{T}\right)$}

Kraft temperatures $\left(\mathrm{k}_{\mathrm{T}}\right)$ of series of surfactants CTAB-1, CTAB-2 and CTAB-3 were measured through conductance measurement as described earlier. The Kraft temperature 
was determined from the plot of specific conductance verses temperature as shown in Figure 2. The conductance was observed to increase rapidly with increase in temperature due to dissolution of the hydrated crystals of surfactant until Kraft temperature reaches. Thereafter the conductance increases slowly due to only increase in mobility of ions with increase in temperature. The Kraft temperature increases from 25 to 27 to $28^{\circ} \mathrm{C}$, when the head group polarity of surfactant increases from CTAB-1, CTAB-2 and CTAB-3 respectively. This can be explained in terms of effect of binding of counterion and head group polarity on micellization and hence on Kraft temperature. It has been reported that increase in hydrophobic alkyl chain length of surfactant molecules assists in micellization and increases the Kraft temperature ${ }^{23}$. This means increase in micellization tendency is related to Kraft temperature. Binding of counterion which usually increases with increase in head group polarity of surfactant would also enhance micellization and hence Kraft temperature.

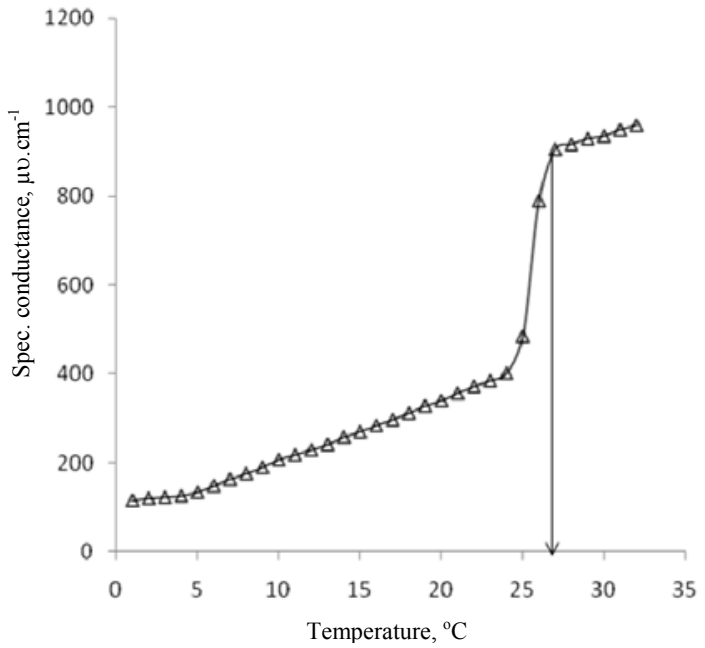

Figure 2. Representative plot for determination of Kraft temperature for CTAB-2 surfactant $(1 \% \mathrm{w} / \mathrm{v}$ solution $)$

\section{Critical micelle concentration (CMC)}

Critical micelle concentration values of surfactant were measured by conductance measurement as function of head group polarity and values is given in Table 1. It is well known that the conductance of surfactant solution changes with concentration at different rates below and above CMC. Surfactants were observed to dissociate completely at very low concentration and their conductance increases linearly with an increase in concentration up to $\mathrm{CMC}$. Although the conductance continues to increase beyond $\mathrm{CMC}$, the rate of increase in conductance is lower compared to that below $\mathrm{CMC}$. This may be due to binding of some of the counterions to the micelles above CMC, causing a reduction in the effective charge on the micelles. The average degree of micelle ionization $(\alpha)$ of a micelle was taken as the ratios of the values of slope $(\mathrm{dk} / \mathrm{dC})$ above and below the CMC. The CMC values of CTAB-1, CTAB-2 and CTAB-3 were observed to decrease from 0.89 to 0.21 to $0.17 \mathrm{mM}$ respectively due to increase in head group polarity. The decrease in average degree of micellar ionization $(\alpha)$ with increase in head group polarity can be attributed to the increase in the polarizability and surface charge density of micelle due to negative inductive effect of $-\mathrm{C}_{2} \mathrm{H}_{4} \mathrm{OH}$ groups and possible intra/inter molecular hydrogen bonding. 
Table 1. CMC, Kraft temperature $\left(\mathrm{k}_{\mathrm{T}}\right)$, Oil (MMA) solubilization, Foamability and Foam stability for cationic surfactants CTAB-1, CTAB-2 and CTAB- 3 at $30{ }^{\circ} \mathrm{C}$

\begin{tabular}{cccccc}
\hline Surfactants & $\begin{array}{c}\mathrm{CMC} \\
\mathrm{mM}\end{array}$ & $\begin{array}{c}\text { Kraft } \\
\text { Temperature } \\
\left(\mathrm{k}_{\mathrm{T}}\right)^{\circ} \mathrm{C}\end{array}$ & $\begin{array}{c}\text { Oil } \\
\text { Solubilization } \\
\mu \mathrm{L}\end{array}$ & $\begin{array}{c}\text { Foamability } \\
\mathrm{cm}^{3}\end{array}$ & $\begin{array}{c}\text { Foam } \\
\text { Stability } \\
\text { Min. }\end{array}$ \\
\hline CTAB-1 & 0.89 & 25 & 150 & 76 & 34 \\
CTAB-2 & 0.21 & 27 & 250 & 56 & 65 \\
CTAB-3 & 0.17 & 28 & 275 & 48 & 75 \\
\hline
\end{tabular}

Foamability and foam stability

Foamability and foam stability of a series of surfactants CTAB-1, CTAB-2 and CTAB-3 $(1 \% \mathrm{w} / \mathrm{v}$ solution) was studied and values are given in Table 1 . In this method foam is produced quickly by rapid shaking of cylinder containing surfactant solution causing a sudden expansion of interfacial area. Foamability was observed to decreases and foam stability was observed to increases as from CTAB-1 to CTAB-3. Foamability and foam stability can be influenced by two independent factors: molecular packing in adsorbed surfactant film at the air/water interface and micelle structure within the bulk water in foam lamellae. This can be explained on the basis of competitive time scales for interfacial area expansion, the diffusion transport of surfactant monomers and the ability of micelle to break up in order to provide monomer flux necessary to stabilize the new air/water interface.

\section{Oil solubilization}

Amount of methyl methacrylate (MMA) solubilized in a micellar solution as function of head group polarity as is given in Figure 3 and values are given in Table 1. The oil solubilisation capacity was observed to increase with increase in head group polarity. This can be attributed to the increase in number of micelles per unit volume and increase in dimension of micelle. This can also be explained by the SANS data as shown in Table 2 .

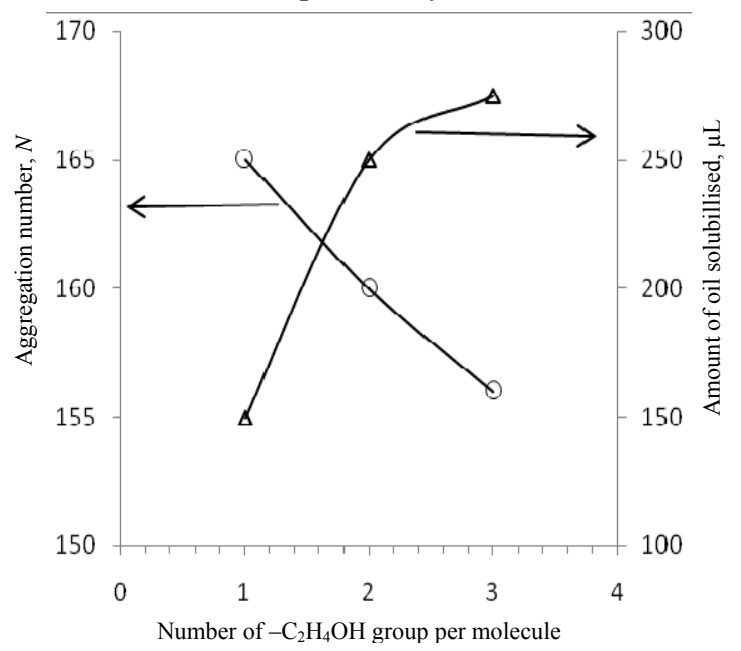

Figure 3. Effect of number of $-\mathrm{C}_{2} \mathrm{H}_{4} \mathrm{OH}$ groups at quaternary nitrogen of cetyl trimethyl ammonium bromide (CTAB) on oil (MMA) solubilizationand aggregation numbers $(\mathrm{N})$ at $30{ }^{\circ} \mathrm{C}$. Oil solubilisation: $(\Delta)$, Aggregation number $(\mathrm{N}):(\mathrm{O})$ 
Table 2. Effect of head group polarity on the micellar parameters of CTAB and its derivatives by SANS at $100 \mathrm{mM}$ concentration and at $30^{\circ} \mathrm{C}$

\begin{tabular}{cccccccc}
\hline Surfactants & $\begin{array}{c}\mathrm{Q}_{\max }, \\
\AA^{-1}\end{array}$ & $\begin{array}{c}\text { Agg. } \\
\text { No. } \\
(\mathrm{N})\end{array}$ & $\begin{array}{c}\text { Fractiona } \\
1 \text { Charge } \\
(\alpha)\end{array}$ & $\begin{array}{c}\text { Semi } \\
\text { major } \\
\text { axis }(\mathrm{b}) \\
(\AA)\end{array}$ & $\begin{array}{c}\text { Semimino } \\
\mathrm{r} \text { axis }(\mathrm{a}) \\
(\AA)\end{array}$ & $\begin{array}{c}\text { Axial } \\
\text { ratio } \\
(\mathrm{b} / \mathrm{a})\end{array}$ & $\begin{array}{c}\text { Equilibriu } \\
\text { m distance } \\
(\mathrm{d})(\AA)\end{array}$ \\
\hline CTAB-1 & 0.05174 & 165 & 0.13 & 50.1 & 21.0 & 2.39 & 7.74 \\
CTAB-2 & 0.04935 & 160 & 0.13 & 52.4 & 21.0 & 2.50 & 7.98 \\
CTAB-3 & 0.04935 & 156 & 0.12 & 55.5 & 21.0 & 2.64 & 8.24 \\
\hline
\end{tabular}

Shape, size and aggregation number

To understand the effect of head polarity on microstructure of surfactant aggregates in aqueous solution, SANS measurements were carried out at $100 \mathrm{mM}$ solution for series of surfactants CTAB-1, CTAB-2 and CTAB-3. SANS distributions as shown in Figure 4, show well defined correlation peaks irrespective of the head group due to the intermicellar structure factor $S(Q)$. The correlation peaks appear at around $Q_{\max } \simeq 2 \pi / D$, where $\mathrm{D}$ is average distance between micelles. Decrease in $\mathrm{Q}_{\max }$ with head group at the same surfactant concentration indicates the increased number density (n) of micelles or decrease in the aggregation number $(\mathrm{N})$. It is observed from Figure 4 that the peak positions slightly shift towards lower $\mathrm{Q}$ values and the peak intensity also decreases slightly for CTAB-3. The observed SANS data were analyzed and micellar parameters such as aggregation number $(\mathrm{N})$, fractional charge $(\alpha)$, semi-minor axis (a), semi-major axis (b) are given in Table 2. The equilibrium distance between the charged heads (d) was calculated ${ }^{24}$ for cationic surfactants under study with respect to head group polarity and is given in Table 2. Aggregation number $(\mathrm{N})$ was decreases and dimension of micelle (b/a) increases with increase in head group polarity. This can be attributed to increase in number of ethanolic groups at quaternary nitrogen of surfactant. The equilibrium distance (d) between the charged head increases with increase in head group polarity, resulting in the increase in the spontaneous packing curvature restricting the growth of micelle subsequently increase the number density of micelle. This can also be attributed by increase in oil solubilization with head group polarity decrease aggregation number $(\mathrm{N})$.

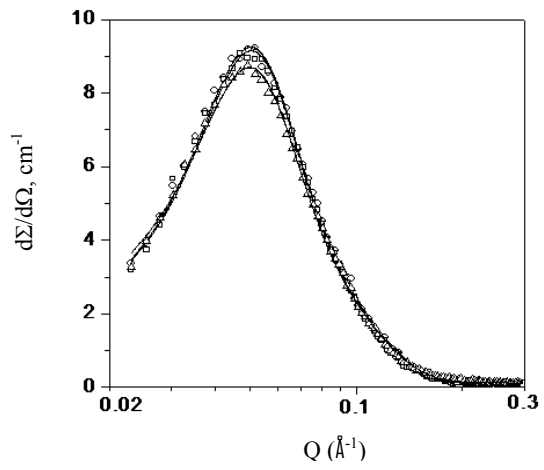

Figure 4. SANS profile for CTAB-1, CTAB-2, CTAB-3 at $100 \mathrm{mM}$ and at $30{ }^{\circ} \mathrm{C}$. The solid line shows the theoretical fits and the symbols are experimentally determined values. CTAB-1 (O), CTAB-2 ( $\square)$ and CTAB-3 $(\Delta)$ 


\section{Conclusion}

The change in molecular architecture of cetyl trimethyl ammonium bromide surfactant by changing head group polarity affects the micellar parameters significantly.

- Critical micellar concentration (CMC) and average degree of micelle ionization $\left(\alpha_{\text {ave }}\right)$ of cationic surfactants significantly decrease with increase in head polarity.

- SANS studies shows that the aggregation number $(\mathrm{N})$ decreases and dimension of micelles (b/a) increases with increase in head polarity.

- The Kraft temperature of surfactant (CTAB) was observed to increases with increase in head group polarity.

- The oil solubilization and foam stability were observed to increases with increase in head group polarity.

\section{Acknowledgement}

Authors are grateful to Dr. V. K. Aswal for providing SANS analysis facility at BARC, Trombay, Mumbai, India.

\section{References}

1. Rosen M J. Surfactants and Interfacial Phenomenon, Second Ed., John Wiley, New York, 1989.

2. Bunton C A, Robinson L, Schaak J and Stern M F, J Org Chem., 1971, 36(16), 2346-2350; DOI:10.1021/jo00815a033

3. Wennerstrom H and Lindman B, Phys Rep., 1979, 52(1), 1-86; DOI:10.1016/0370-1573(79)90087-5

4. Menger F M, Angew Chem Int Ed Engl., 1991, 30(9), 1086-1099; /DOI:10.1002/anie.199110861

5. Zana R, Benrroau M and Rueff R, Langmuir, 1991, 7(6), 1072-1075; DOI:10.1021/la00054a008

6. $\quad$ Rosen M J, Mathias J H and Davenport L, Langmuir, 1999, 15(21), 7340-7346; DOI:10.1021/la9904096

7. $\quad$ Song L D and Rosen M J, Langmuir, 1996, 12(5), 1149-1153; DOI:10.1021/la950508t

8. Wettig S D and Verrall R E, J Colloid Interface Sci., 2001, 235(2), 310-316; DOI:10.1006/jcis.2000.7348

9. $\quad$ Chen S H, Annu Rev Phys Chem., 1986, 37, 351-399.

10. Aswal V K and Goyal P S, Curr Sci., 2000, 79, 947.

11. Goyal P S, Phase Transit., 1994, 50(1-3), 143-176; DOI:10.1080/01411599408200372

12. Adamczyk Z, Para G and Warszynski P, Langmuir, 1999, 15(24), 8383-8387; DOI:10.1021/la990241o

13. Bakshi M S, Colloid Polym Sci., 2000, 278(12), 1155-1163.

14. Skerjanc J, Kogej K and Cerar J, Langmuir, 1999, 15(15), 5023-5028; DOI:10.1021/la981710+

15. Zielinski R, J Colloid Interface Sci., 2001, 235(2), 201-209; DOI:10.1006/jcis.2000.7364

16. Ranganathan R, Okano L T, Yihwa C and Quina F H, J Colloid Interface Sci., 1999, 214(2), 238-242; DOI:10.1006/jcis.1999.6217

17. Thomas H G, Lomakin A, Blankschtein D and Benedek G B, Langmuir, 1997, 13(2), 209-218; DOI:10.1021/1a9606613 
18. Zana R, Adv Colloide Interface Sci., 2002, 97(1-3), 205-253;

DOI:10.1016/S0001-8686(01)00069-0

19. Mukerjiee P and Mysels K J, Critical Micellar Concentrations of Aqueous Surfactant Systems, National Bureau of Standards, Washington, DC, USA, 1971.

20. Borse M, Sharma V, Aswal V K, Pokhariyal N K, Joshi J V, Goyal P S and Surekha D, Phys Chem Chem Phys., 2004, 6, 3508-3514; DOI:10.1039/B402767C

21. Shah D O, J Colloid Interface Sci., 1971, 37(4), 744-762;

DOI:10.1016/0021-9797(71)90353-5

22. Patist A, Devi S and Shah D O. Langmuir, 1999, 15(21), 7403-7405;

DOI:10.1021/la9901128

23. Zana R, Adv Colloid Interface Sci., 2002, 97, 203.

24. Halder J, Aswal V K, Goyal P S and Bhattacharya S, Angew Chem., Int Ed., 2001, 40(7), 1228-1232;

DOI:10.1002/1521-3773(20010401)40:7<1228::AID-ANIE1228>3.0.CO;2-I 